O. IKAWA

KODAI MATH. J.

19 (1996), $168-176$

\title{
YANG-MILLS HOMOGENEOUS CONNECTIONS ON COMPACT SIMPLE LIE GROUPS
}

\author{
Dedicated to Tsunero Takahashi on his 60 's birthday
}

OSAMU IKAWA

\section{Introduction}

Let $M$ be a compact Riemannian manifold and $P$ a principal $G$-bundle, where $G$ is a compact Lie group. Fix a bi-invariant Riemannian metric on $G$. Let $\Omega_{\Lambda}$ denote the curvature form of a connection $\Lambda$ on $P$. A critical point of the Yang-Mills functional

$$
\Lambda \mapsto \frac{1}{2} \int_{M}\left\|\Omega_{\Lambda}\right\|^{2}
$$

is called a Yang-Mills connection. A Yang-Mills connection $\Lambda$ is said to be stable if the second variation of the Yang-Mills functional is non-negative. A flat connection is a stable Yang-Mills connection. H. T. Laquer [4] proved that (0)-connection on a compact Lie group is an unstable Yang-Mills connection. A compact Riemannian manifold $M$ is called Yang-Mills unstable if, for every choice of $G$ and every principal $G$-bundle $P$ over $M$, stable Yang-Mills connection is always flat. S. Kobayashi, Y. Ohnita and M. Takeuchi [3] classified the compact simply connected irreducible symmetric spaces of type I which are Yang-Mills unstable. In their paper, they gave a following question :

Is every simply connected compact simple Lie group Yang-Mills unstable? In this paper, we consider an equivariant $G$-bundle $P$ over a compact connected simple Lie group $L$. It is obtained by a Lie homomorphism $\rho: L \rightarrow G$. With respect to homogeneous connections on $P$, we get the following:

THEOREM 1. Consider the following three conditions (1), (2), and (3):

(1) $\rho$ is indecomposable (see $\S 2$ for definition),

(2) Flat homogeneous connections are only ( \pm )-connections,

(3) (0)-connection is a unique non-flat Yang-Mills homogeneous connection.

Then (1) and (2) are equivalent. (3) implies (1).

Received January 11, 1995; revised May 29, 1995. 
Moreover if $\rho(\mathfrak{l})$ contains a regular element of $g$, then (1) implies (3). In general, (1) does not imply (3) (see $\S 3$ ).

THEOREM 2. Assume $\rho(\mathfrak{l})$ contains a regular element of $\mathfrak{g}$. Then any nonflat Yang-Mills homogeneous connection is unstable.

\section{Proof of theorems}

Let $L$ be a compact connected simple Lie group with Lie algebra $\mathfrak{l}$. Take an $\operatorname{Ad}(L)$-invariant inner product $\langle$,$\rangle on \mathfrak{l}$. Let $G$ be an another compact connected Lie group with Lie algebra g. Take an $\operatorname{Ad}(G)$-invariant inner product $\langle$,$\rangle on g$. Let $\rho: L \rightarrow G$ be a Lie homomorphism. We denote the differential Lie homomorphism of $\rho$ by the same symbol $\rho$. Put

$$
K=L \times L \supset H=\{(l, l) ; l \in L\} \cong L((l, l) \leftrightarrow l) \text { and } M=K / H .
$$

We define an inner product $\langle$,$\rangle on \mathfrak{f}$ by

$$
\langle(X, Y),(Z, W)\rangle=2(\langle X, Z\rangle+\langle Y, W\rangle) \text { for } X, Y, Z, W \in \mathfrak{l} .
$$

We define an $\operatorname{Ad}(H)$-invariant subspace $\mathfrak{m}$ of $\mathfrak{f}$ by

$$
\mathfrak{m}=\{(X,-X) ; X \in \mathfrak{l}\} .
$$

Then we have:

$$
\mathfrak{f}=\mathfrak{h}+\mathfrak{m} \quad \text { (direct sum). }
$$

The induced $\operatorname{Ad}(H)$-invariant inner product on $m$ naturally induces a $K$-invariant Riemannian metric on $M$. The mapping

$$
(a, b) H \mapsto a b^{-1}
$$

is an isometry from $M$ onto $L$. The mapping

$$
\mathfrak{m} \rightarrow \mathfrak{l} ; \quad\left(\frac{1}{2} X,-\frac{1}{2} X\right) \mapsto X
$$

is a linear isometry from $\mathfrak{m}$ onto $\mathfrak{l}$. In this correspondence, we have

$$
(\operatorname{Ad}(H), \mathfrak{m}) \cong(\operatorname{Ad}(L), \mathfrak{l}) .
$$

We define a Lie homomorphism $\bar{\rho}$ from $H$ into $G$ by

$$
\bar{\rho}: H \rightarrow G ;(l, l) \mapsto \rho(l) .
$$

Every Lie homomorphism from $H$ into $G$ is obtained in this way. The space of homogeneous connections on the principal $G$-bundle $P=K \times_{\bar{\rho}} G$ over $M$ is identified with

$$
\operatorname{Hom}_{L}(\mathfrak{l}, \mathfrak{g})=\{\Lambda \in \operatorname{Hom}(\mathfrak{l}, \mathfrak{g}) ;[\rho(X), \Lambda(Y)]=\Lambda([X, Y]) \text { for } X, Y \in \mathfrak{l}\} .
$$


by Wang's theorem ([2, pp. 106-107, Theorem 11.5]), where $\operatorname{Hom}(\mathfrak{l}, \mathfrak{g})$ is the space of linear mappings from the vector space $\mathfrak{l}$ to the vector space $\mathfrak{g}$. Remark that $\boldsymbol{R} \rho$ is contained in $\operatorname{Hom}_{L}(\mathfrak{l}, \mathfrak{g})$. The curvature from $\Omega$ of a homogeneous connection $\Lambda \in \mathrm{Hom}_{L}(\mathfrak{l}, \mathfrak{g})$ is an alternative linear mapping from $\mathfrak{l} \times \mathfrak{l}$ to $\mathfrak{g}$ which is given by

$$
2 Q(X, Y)=-\frac{1}{4} \rho([X, Y])+[\Lambda(X), \Lambda(Y)] .
$$

In particular, the curvature form $\Omega_{t}$ of $t \rho \in \boldsymbol{R} \rho$ is

$$
2 \Omega_{t}(X, Y)=\left(t^{2}-\frac{1}{4}\right) \rho([X, Y]) .
$$

Hence $\Lambda=( \pm 1 / 2) \rho$ are flat connections, which are called $( \pm)$-connection, respectively. A critical point of the Yang-Mills functional $\Lambda \mapsto\|\Omega\|^{2}$ is called a Yang-Mills connection. A homogeneous connection $\Lambda \in \mathrm{Hom}_{L}(\mathfrak{l}, \mathfrak{g})$ is Yang-Mills if and only if for each $X \in \mathfrak{l}$

$$
\sum_{\imath=1}^{n}\left[\Lambda\left(E_{\imath}\right), \Omega\left(E_{\imath}, X\right)\right]=0,
$$

where $\left\{E_{1}, \cdots, E_{n}\right\}$ is an orthonormal basis of $\mathfrak{l}$. In particular, $\Lambda=0$ is a YangMills connection, which is called the (0)-connection.

DeFINITION 1. We say that $\rho$ is indecomposable, if

$$
\begin{aligned}
& \rho=\rho_{1}+\rho_{2}, \rho_{\imath}: \mathfrak{l} \rightarrow \mathfrak{g}: \text { Lie homomorphism s.t. }\left[\operatorname{Im} \rho_{1}, \operatorname{Im} \rho_{2}\right]=0(*) \\
& \Rightarrow \rho_{1}=0, \rho_{2}=\rho \text { or } \rho_{2}=0, \rho_{1}=\rho .
\end{aligned}
$$

We say that $(*)$ is a decomposition of $\rho$.

Since the kernel of $\rho$ is an ideal of $\mathfrak{l}, \rho$ is injective or $\rho=0$. If $\rho=0$, then $\operatorname{Hom}_{L}(\mathfrak{l}, \mathfrak{g})=\{0\}$ and $(0)$-connection is flat. Therefore we may assume that $\rho$ is injective.

THEOREM 1. Consider the following three conditions (1), (2), and (3):

(1) $\rho$ is indecomposable,

(2) Flat homogeneous connections are only the (士)-connections,

(3) The (0)-connection is a unique non-flat Yang-Mills homogeneous connection.

Then (1) and (2) are equivalent. The condition (3) implies (1). Moreover if $\rho(\mathfrak{l})$ contains a regular element of $\mathfrak{g}$, then (1) implies (3).

Remark 1. In general, (1) does not imply (3) (see §3).

Proof of the first half of Theorem 1. If $\rho=\rho_{1}+\rho_{2}$ is a non-trivial decomposition of $\rho$, then $1 / 2\left(\rho_{1}-\rho_{2}\right)$ is a flat homogeneous connection except the $( \pm)$-connection and $(1 / 2) \rho_{1}$ is a non-flat Yang-Mills connection except the 
(0)-connection. Hence (2) implies (1), and (3) implies (1). We show (1) implies (2). Let $\Lambda$ be any flat homogeneous connection. Put

$$
\rho_{1}=\frac{1}{2} \rho+\Lambda, \quad \rho_{2}=\frac{1}{2} \rho-\Lambda .
$$

Then $\rho=\rho_{1}+\rho_{2}$ is a decomposition of $\rho$. Since $\rho$ is indecomposable, $\rho_{1}=0$ or $\rho_{1}=\rho$. Hence $\Lambda=( \pm 1 / 2) \rho$.

THEOREM 2. Assume $\rho(\mathfrak{l})$ contains a regular element of $\mathrm{g}$. Then any nonflat Yang-Mills homogeneous connection is unstable.

Proof of the second half of Theorem 1 and Theorem 2. It is sufficient to prove that for each non-flat Yang-Mills connection $\Lambda \in \mathrm{Hom}_{L}(\mathfrak{l}, \mathfrak{g})$, there exists $\alpha\left(=\alpha_{A}\right) \in \operatorname{Hom}_{L}(\mathfrak{l}, \mathfrak{g})$ such that

(A1) $\alpha=0$ implies $\Lambda=0$,

(A2) $\rho=\alpha+(\rho-\alpha)$ is a decomposition of $\rho$, and $\rho-\alpha \neq 0$,

(A3) $d^{2} / d t^{2}\left\|\Omega_{t}\right\|_{1 t=0}<0$, where $\Omega_{t}$ is the curvature form of $\Lambda+t(\rho-\alpha)$.

Applying Whitehead's vanishing theorem of cohomology group ( $[6$, p. 95, Theorem 13]) for the representation $(\operatorname{ad} \circ \rho, \mathfrak{g})$ of $\mathfrak{l}$, we have following:

If $\Lambda_{1}, \Lambda_{2} \in \operatorname{Hom}_{L}(\mathfrak{l}, \mathfrak{g})$ satisfy

(B1) $\left[\Lambda_{1}(X), \Lambda_{2}(Y)\right]=-\left[\Lambda_{1}(Y), \Lambda_{2}(X)\right]$,

(B2) $\varsigma_{X, Y, Z}\left[\rho(X),\left[\Lambda_{1}(Y), \Lambda_{2}(Z)\right]\right]=0$, where $\varsigma_{X, Y, Z}$ is the sum over the cyclic permutations of $X, Y, Z$,

then there exists $\Lambda_{3} \in \operatorname{Hom}_{L}(\mathfrak{l}, \mathfrak{g})$ such that

$$
\left[\Lambda_{1}(X), \Lambda_{2}(Y)\right]=\Lambda_{3}([X, Y]) .
$$

Remark that under the condition (B1), the condition (B2) is equivalent to $\Im_{X, Y, Z}\left[\Lambda_{1}([X, Y]), \Lambda_{2}(Z)\right]=0$. Since $\rho(\mathfrak{l})$ contains a regular element of $\mathfrak{g}$, $\left[\Lambda_{1}, \Lambda_{2}\right]$ is skew-symmetric automatically. In fact, take Cartan subalgebras $t$ and $\mathfrak{h}$ of $\mathfrak{l}$ and $g$ respectively such that $\rho(\mathfrak{t}) \subset \mathfrak{h}$. Then

$$
\left[\rho(\mathrm{t}), \Lambda_{i}(\mathrm{t})\right]=\Lambda_{i}([\mathrm{t}, \mathrm{t}])=0 .
$$

This implies $\Lambda_{i}(\mathrm{t}) \subset \mathrm{h}$ by assumption. In particular, $\left[\Lambda_{1}(\mathrm{t}), \Lambda_{2}(\mathrm{t})\right]=0$ and $\left[\Lambda_{1}(H), \Lambda_{2}(H)\right]=0$ for $H \in \mathrm{t}$. Since $\mathfrak{l}=\operatorname{Ad}(L) \mathfrak{t}([1$, p. 248, Theorem 6.4]), we get $\left[\Lambda_{1}(X), \Lambda_{2}(X)\right]=0$.

Let $\Lambda \in \operatorname{Hom}_{L}(\mathfrak{l}, \mathfrak{g})$ be any non-flat Yang-Mills homogeneous connection. First we prove $\Im_{X, Y, Z}[\rho(X),[\Lambda(Y), \Lambda(Z)]]=0$ using the classification of compact simple Lie algebras. The vector space

$$
V=\mathfrak{l} \wedge \mathfrak{l}=\operatorname{span}\{X \wedge Y ; X, Y \in \mathfrak{l}\}
$$

is an $\mathfrak{l}$-module by the $\mathfrak{l}$-action: 


$$
(\operatorname{ad} Z)(X \wedge Y)=[Z, X] \wedge Y+X \wedge[Z, Y] \text {. }
$$

The space

$$
W=\operatorname{span}\{[\Lambda(X), \Lambda(Y)] ; X, Y \in \mathfrak{l}\}
$$

is an $\operatorname{ad}(\rho(\mathfrak{l}))$-invariant subspace of $\mathfrak{g}$. We consider the $\mathfrak{l}$-homomorphism $\Phi$ from $V$ onto $W$ which is defined by

$$
\Phi: V=\mathfrak{l} \wedge \mathfrak{l} \rightarrow W ; X \wedge Y \mapsto[\Lambda(X), \Lambda(Y)] .
$$

Since $\Phi$ is surjective, $V / V_{0} \cong W$ as $\mathfrak{l}$-modules, where $V_{0}=\operatorname{Ker} \Phi$. On the other hand, we consider the $\mathfrak{l}$-homomorphism $\Psi$ from $V$ into $\mathfrak{l}$ which is defined by

$$
\Psi: V=\mathfrak{l} \wedge \mathfrak{l} \rightarrow \mathfrak{l} ; X \wedge Y \leftrightarrow[X, Y] .
$$

Since $[\mathfrak{l}, \mathfrak{l}]=\mathfrak{l}, \Psi$ is surjective. We show that the irreducibility of $V_{1}=\operatorname{Ker} \Psi$. We denote by $\mathfrak{l}^{c}, \mathrm{t}^{c}$ and $\rho^{c}$ the complexifications of $\mathfrak{l}, \mathrm{t}$ and $\rho$ respectively. The complex Lie algebra $\mathfrak{l}^{C}$ is simple. We denote by $\Delta$ the set of nonzero roots of $\mathfrak{l}^{c}$ with respect to $\mathfrak{t}^{c}$. For $\alpha \in \Delta$, there exists a non-zero vector $E_{\alpha} \in \mathfrak{l}^{c}$ such that

$$
\left[H, E_{\alpha}\right]=\alpha(H) E_{\alpha} \text { for all } H \in \mathrm{t}^{C} .
$$

We have a direct-sum decomposition :

$$
\mathfrak{l}^{C}=\mathrm{t}^{C}+\sum_{\alpha \in \Delta} C E_{\alpha} .
$$

Fix a lexicographic ordering on $t^{C}$. We denote by $\delta_{0}$ the highest root of $\Delta$ and by $\left\{\alpha_{1}, \cdots, \alpha_{r}\right\}$ the set of simple roots of $\Delta$. The set

$$
\left\{\delta_{0}-\alpha_{i} \in \Delta\right\} \neq \emptyset
$$

is a single point set $\left\{\delta_{1}\right\}$ or two points set $\left\{\delta_{1}, \delta_{2}\right\}$, and the set consists two points if and only if $\mathfrak{l}=\mathfrak{g} \mathfrak{u}(m)$.

In the case where $\left\{\delta_{0}-\alpha_{i} \in \Delta\right\}=\left\{\delta_{1}\right\}$, we define an $\mathfrak{l}$-invariant subspace $V_{1}\left(\delta_{0}+\delta_{1}\right)$ of $V_{1}^{c}$ by

$$
V_{1}\left(\delta_{0}+\delta_{1}\right)=\operatorname{ad}\left(U\left(\mathfrak{l}^{C}\right)\right)\left(E_{\delta_{0}} \wedge E_{\delta_{1}}\right),
$$

where $U\left(\mathfrak{l}^{c}\right)$ is the universal enveloping algebra of $\mathfrak{l}^{c}$. The highest weight of $V_{1}\left(\delta_{0}+\delta_{1}\right)$ is $\delta_{0}+\delta_{1}$ and the multiplicity of $\delta_{0}+\delta_{1}$ is equal to 1 . Hence $V_{1}\left(\delta_{0}+\delta_{1}\right)$ is irreducible. By virtue of Weyl's dimensionality formula $([6$, p. 257]), we get

$$
\operatorname{dim} V_{1}\left(\boldsymbol{\delta}_{0}+\boldsymbol{\delta}_{1}\right)=\frac{\operatorname{dim} \mathfrak{l}(\operatorname{dim} \mathfrak{l}-3)}{2}=\operatorname{dim} V_{1} .
$$

Hence $V_{1}^{C}=V_{1}\left(\boldsymbol{\delta}_{0}+\boldsymbol{\delta}_{1}\right)$. In particular, $V_{1}^{C}$ is irreducible so $V_{1}$ is.

In the case where $\left\{\delta_{0}-\alpha_{i} \in \Delta\right\}=\left\{\delta_{1}, \delta_{2}\right\}$, we define $\mathfrak{l}$-invariant subspaces $V_{1}\left(\delta_{0}+\delta_{1}\right)$ and $V_{1}\left(\delta_{0}+\delta_{2}\right)$ of $V_{1}^{c}$ by 


$$
\begin{aligned}
& V_{1}\left(\boldsymbol{\delta}_{0}+\boldsymbol{\delta}_{1}\right)=\operatorname{ad}\left(U\left(\mathfrak{l}^{c}\right)\right)\left(E_{\delta_{0}} \wedge E_{\delta_{1}}\right), \\
& V_{1}\left(\delta_{0}+\delta_{2}\right)=\operatorname{ad}\left(U\left(\mathfrak{l}^{c}\right)\right)\left(E_{\delta_{0}} \wedge E_{\delta_{2}}\right) .
\end{aligned}
$$

For $i=1,2$, the highest weight of $V_{1}\left(\boldsymbol{\delta}_{0}+\boldsymbol{\delta}_{i}\right)$ is $\delta_{0}+\boldsymbol{\delta}_{i}$ and the multiplicity of $\delta_{0}+\delta_{i}$ is equal to 1 Hence $V_{1}\left(\delta_{0}+\delta_{i}\right)(i=1,2)$ is irreducible. By virtue of Weyl's dimensionality formula, we get

$$
\operatorname{dim} V_{1}\left(\boldsymbol{\delta}_{0}+\boldsymbol{\delta}_{1}\right)=\operatorname{dim} V_{1}\left(\boldsymbol{\delta}_{0}+\boldsymbol{\delta}_{2}\right)=\frac{1}{2} \operatorname{dim} V_{1} .
$$

Hence we have

$$
V_{1}^{C}=V_{1}\left(\delta_{0}+\delta_{1}\right)+V_{1}\left(\delta_{0}+\delta_{2}\right) \text { (direct sum). }
$$

We denote by $W(L)$ the Weyl group of $L$. Clearly, there exist $\sigma_{1}, \sigma_{2} \in W(L)$ such that

$$
\sigma_{1}\left(\delta_{0}+\delta_{1}\right)=-\left(\delta_{0}+\delta_{2}\right), \quad \sigma_{2}\left(\delta_{0}+\delta_{2}\right)=-\left(\delta_{0}+\delta_{1}\right) .
$$

Hence $V_{1}$ is real irreducible, whether $\left\{\delta_{0}-\alpha_{i} \in \Delta\right\}$ is a single point set or two points set. So we get

$$
V_{1}=\operatorname{ad}(U(\mathfrak{l}))(\mathrm{t} \wedge \mathrm{t}) \subset V_{0} .
$$

Hence $\Phi$ naturally induces $\mathfrak{l}$-homomorphism $\varphi$ from $V / V_{1}$ onto $W$ defined by

$$
\varphi: V / V_{1} \rightarrow W ; \overline{X \wedge Y} \mapsto[\Lambda(X), \Lambda(Y)],
$$

where $\overline{X \wedge Y}$ is the equivalence class of $X \wedge Y$. From Jacobi's identity, we have

$$
\begin{aligned}
\Im_{X, Y, Z} \operatorname{ad}(Z) \overline{X \wedge Y} & =\Im_{X, Y, Z}(\overline{[Z, X] \wedge Y+X \wedge[Z, Y]}) \\
& =2 \Im_{X, Y, Z} \overline{[Z, X] \wedge Y} \\
& =0 .
\end{aligned}
$$

Hence we have

$$
0=\varphi\left(\Im_{X, Y, Z} \operatorname{ad}(Z) \overline{X \wedge Y}\right)=\Im_{X, Y, Z}[\rho(Z),[\Lambda(X), \Lambda(Y)]] .
$$

By Whitehead's vanishing theorem of cohomology group, there exists $\alpha \in$ $\operatorname{Hom}_{L}(\mathfrak{l}, g)$ such that

$$
\alpha([X, Y])=4[\Lambda(X), \Lambda(Y)] .
$$

By Jacobi's identity, we have

$$
\mathfrak{\Im}_{X, Y, Z}[\alpha([X, Y]), \Lambda(Z)]=\frac{1}{4} \mathfrak{\varsigma}_{X, Y, Z}[[\Lambda(X), \Lambda(Y)], \Lambda(Z)]=0 .
$$

By Whitehead's vanishing theorem of cohomology group, there exists $\Gamma \in$ $\operatorname{Hom}_{L}(\mathfrak{l}, \mathfrak{g})$ such that

$$
[\alpha(X), \Lambda(Y)]=\Gamma([X, Y])
$$


Since $\Lambda$ is Yang-Mills, we have

$$
\begin{aligned}
-\frac{c}{4} \Gamma(X) & =\frac{1}{4} \sum_{\imath=1}^{n}\left[\Lambda\left(E_{\imath}\right), \alpha\left(\left[E_{\imath}, X\right]\right)\right] \\
& =\sum_{\imath=1}^{n}\left[\Lambda\left(E_{\imath}\right),\left[\Lambda\left(E_{\imath}\right), \Lambda(X)\right]\right] \\
& =-\frac{c}{4} \Lambda(X),
\end{aligned}
$$

where $c$ is the eigenvalue of the negative of the Casimir operator of $(\mathrm{ad}, \mathfrak{l})$. Hence $\Gamma=\Lambda$, that is,

$$
[\alpha(X), \Lambda(Y)]=\Lambda([X, Y]) .
$$

Hence we get (A1). We show $\alpha$ is a Lie homomorphism. From Jacobi's identity, we have

$$
\begin{aligned}
\frac{1}{4}[\alpha(X), \alpha([Z, W])] & =[\alpha(X),[\Lambda(Z), \Lambda(W)]] \\
& =[[\alpha(X), \Lambda(Z)], \Lambda(W)]+[\Lambda(Z),[\alpha(X), \Lambda(W)]] \\
& =[\Lambda([X, Z]), \Lambda(W)]+[\Lambda(Z),[\Lambda([X, W])]] \\
& =\frac{1}{4} \alpha([[X, Z], W]+[Z,[X, W]]) \\
& =\frac{1}{4} \alpha([X,[Z, W]]) .
\end{aligned}
$$

Hence $\alpha \in \operatorname{Hom}_{L}(\mathfrak{l}, \mathfrak{g})$ is a Lie homomorphism. So, if we put $\delta=\rho-\alpha$, then $\rho=\alpha+\delta$ is a decomposition of $\rho$. The curvature form $\Omega$ of $\Lambda$ is given by $\Omega(X, Y)=$ $(-1 / 4) \delta([X, Y])$. Since $\Lambda$ is not flat, we have $\delta \neq 0$. Hence we have (A2). Since $[\delta(X), \Lambda(Y)]=0$, the curvature form $\Omega_{t}$ of $\Lambda+t \delta$ is given by

$$
\Omega_{t}(X, Y)=\frac{4 t^{2}-1}{4} \delta([X, Y])
$$

Hence we have (A3).

\section{An example}

When $\rho(\mathfrak{l})$ does not contain any regular element of $\mathfrak{g}$, the $(0)$-connection is not necessarily a unique non-flat Yang-Mills homogeneous connection, even if $\rho$ is indecomposable. We show such an example. Put $L=S U(m)$ for $m \geqq 3$. We define an $\operatorname{Ad}(L)$-invariant inner product $\langle$,$\rangle on \mathfrak{l}$ by

$$
\langle X, Y\rangle=-\operatorname{tr}(X Y) \text { for } X, Y \in \mathfrak{l} \text {. }
$$

The inner product $\langle$,$\rangle naturally induces a Hermitian inner product \langle$, $\rangle$ on $\mathfrak{l}^{c}$. Put $G=S U\left(\mathfrak{I}^{c}\right)$ and $\rho=\mathrm{Ad}: L \rightarrow G$. In this case, $\rho(\mathfrak{l})$ does not contain any 
regular element of $\mathfrak{g}$. We define an $\operatorname{Ad}(G)$-invariant inner product $\langle$,$\rangle on \mathfrak{g}$ by

$$
\langle A, B\rangle=\sum_{\imath=1}^{m^{2}-1}\left\langle A E_{\imath}, B E_{\imath}\right\rangle \text { for } A, B \in \mathrm{g},
$$

where $\left\{E_{i}\right\}_{1 \leqq \imath \leqq m^{2}-1}$ is an orthonormal basis of $\mathfrak{l}$. We define a homogeneous connection $\Lambda \in \operatorname{Hom}_{L}(\mathfrak{l}, \mathrm{g})$ by

$$
(\Lambda(X))(Y)=\frac{-m}{2 \sqrt{m^{2}+4}}\left\{(X Y+Y X)-\frac{2}{m} \operatorname{tr}(X Y) 1_{m}\right\},
$$

where $1_{m}$ is the identity matrix (cf. [5]).

Remark 2. If $m=2$, then $\Lambda=0$.

Proposition 1. (1) $\operatorname{Hom}_{L}(\mathfrak{l}, \mathfrak{g})=\boldsymbol{R} \rho+\boldsymbol{R} \Lambda$ (orthogonal direct sum),

(2) $\rho$ is indecomposable,

(3) $\Lambda(\neq 0)$ is a non-flat Yang-Mills homogeneous connection, which is a local minimum on the space of homogeneous connections $\operatorname{Hom}_{L}(\mathfrak{l}, \mathfrak{g})$.

Proof. (1) is obtained by simple calculation. (2) is obtained by (1) and Theorem 1.

(3) The equations

$$
\sum_{\imath=1}^{m^{2}-1}\left[E_{\imath},\left[E_{\imath}, X\right]\right]=-2 m X, \quad \sum_{\imath=1}^{m^{2}-1} E_{\imath}^{2}=-\frac{m^{2}-1}{m} 1_{m}
$$

and

$$
\begin{aligned}
& {[\Lambda(X),[\Lambda(Y), \Lambda(Z)]](W)} \\
& =\frac{m^{2}}{4\left(m^{2}+4\right)} \Lambda([X,[Y, Z]])(W) \\
& \quad+\frac{m}{m^{2}+4}\{\operatorname{tr}(Y W) \Lambda(X) Z-\operatorname{tr}(Z W) \Lambda(X) Y \\
& \quad-\operatorname{tr}(Y \Lambda(X) W) Z+\operatorname{tr}(Z \Lambda(X) W) Y\}
\end{aligned}
$$

imply that $\Lambda$ is a non-flat Yang-Mills homogeneous connection.

Put $\Lambda(x, y)=(x / 2) \rho+y \Lambda$ and $f(x, y)=4\|\Omega(x, y)\|^{2}$, where $\Omega(x, y)$ is the curvature form of $\Lambda(x, y)$. The equations

$$
\begin{aligned}
& \sum_{\imath, j}\left\|\rho\left(\left[E_{\imath}, E_{\jmath}\right]\right)\right\|^{2}=4 m^{2}\left(m^{2}-1\right), \\
& \sum_{\imath, j}\left\|\Lambda\left(\left[E_{\imath}, E_{\jmath}\right]\right)\right\|^{2}=\frac{m^{2}\left(m^{2}-1\right)\left(m^{2}-4\right)}{m^{2}+4}, \\
& \sum_{\imath, j}\left\|\left[\Lambda\left(E_{\imath}\right), \Lambda\left(E_{j}\right)\right]\right\|^{2}=\frac{m^{2}\left(m^{2}-1\right)\left(m^{2}-4\right)}{4\left(m^{2}+4\right)}
\end{aligned}
$$

imply that 


$$
\begin{aligned}
f(x, y)=m^{2}\left(m^{2}-1\right)\{ & \frac{1}{4}\left(x^{2}-1\right)^{2}+\frac{m^{2}-4}{4\left(m^{2}+4\right)} y^{4} \\
& \left.+\frac{m^{2}-4}{m^{2}+4} x^{2} y^{2}+\frac{m^{2}-4}{2\left(m^{2}+4\right)}\left(x^{2}-1\right) y^{2}\right\} .
\end{aligned}
$$

Hence $f$ is a local minimum at $(0,1)$.

\section{References}

[1] S. Helgason, Differential Geometry, Lie Groups and Symmetric Spaces, Academic Press, New York-San Francisco-London, 1978.

[2] S. Kobayashi and K. Nomizu, Foundations of Differential Geometry I, Interscience Publishers, 1963.

[3] S. Kobayashi, Y. Ohnita and M. Takeuchi, On instability of Yang-Mills connections, Math. Z., 193 (1986), 165-189.

[4] H.T. LAQUER, Stability properties of the Yang-Mills functional near the canonical connection, Michigan Math. J., 31 (1984), 139-159.

[5] H.T. LAQUER, Invariant affine connections on Lie groups, Trans. Amer. Math. Soc., 331 (1992), 541-551.

[6] N. JacoBson, Lie Algebras, Dover Publications, 1962.

Department of Mathematics

Fukushima National College of Technology

FUKUSHIMA, 970

JAPAN 